\title{
Experimental results and basic considerations concerning injection and transport of electrons in the dye-sensitized colloidal sponge-type anatase $\mathrm{TiO}_{2}$ electrode
}

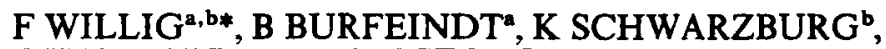 \\ T HANNAPPEL ${ }^{\mathrm{a}}$, and $\mathrm{W}$ STORCK ${ }^{\mathrm{a}}$ \\ -Fritz-Haber-Institut der Max-Planck-Gesellschaft, Faradayweg 4-6, 14195 \\ Berlin, Germany \\ ${ }^{6}$ Hahn-Meitner-Institut, Glienickerstr. 100, 14109-Berlin, Germany
}

\begin{abstract}
Electron transfer over the reaction distance corresponding to three saturated bonds occurs within 70 fs from the excited singlet state of anchored perylene chromophores to empty electronic levels in a sponge-type anatase $\mathrm{TiO}_{2}$ electrode. Injected electrons can be consumed in three different types of recombination processes at the interface with the electrolytic contact. Electron transport through the electrode follows a potential gradient that is set-up in the dark, corresponding to the difference between the Fermi levels of the two contact materials. It is dominated by multiple trapping and trap filling processes.
\end{abstract}

Keywords. Colloidal sponge-type $\mathrm{TiO}_{2}$ electrode; multiple trapping; electrolytic contact; anchored perylene chromophores.

\section{Introduction}

This paper addresses basic features of dye-sensitized electron injection occuring in a colloidal sponge-type $\mathrm{TiO}_{2}$ electrode upon illumination with visible light. Recently, several laboratories have made an increasing effort towards understanding details of this process. At the same time the Lausanne group ${ }^{1}$ and associated laboratories have reported significant progress towards utilizing this effect for the construction of a commercial solar cell.

The basic effect of dye sensitized electron injection into a wide band gap semiconductor is known since a long time. Dye sensitized current flow in semiconductors was reported repeatedly in the past for solid state systems ${ }^{2}$ and electrochemical systems ${ }^{3}$. The effect has been utilized, since the end of the last century, for carrying out silver-halide photography ${ }^{4}$. For the latter process, electron transfer was considered a possible primary reaction mechanism along with other scenarios ${ }^{4}$. However, it turned out that picosecond to femtosecond time resolution was required for the time-resolved observation of the light-driven interfacial electron transfer reaction in this system. Measurements with sufficient time-resolution that are not affected by the irreversible nature of the photographic process have become available only recently 5 .

Efficient absorption of all the visible light by a monomolecular dye layer turned out to be one of the crucial requirements for applying dye-sensitized electron injection for the construction of a solar cell. Generally, a thick dye layer cannot be applied since most dye layers show a very short diffusion length of the excited state, a few hundred

*For correspondence 
angstroms at the most, for a perfectly ordered molecular layer ${ }^{6}$. Essential for achieving efficient light absorption is therefore a tremendously folded or corrugated surface area of the semiconductor electrode, where the imping light can pass many times through different sections of the folded monomolecular dye layer ${ }^{7-9}$. Another important requirement is the use of appropriate dye molecules with a broad absorption spectrum that can absorb all the visible light ${ }^{10}$.

There are additional kinetic requirements to be met for the functioning of such a dye-sensitized semiconductor electrode as an efficient solar cell. Experimental results and basic considerations are presented in this paper that address two essential kinetic features of the system, i.e. electron injection at the interface and electron transport through the electrode. It is obvious that electron injection must be fast compared to the other decay channels available to the excited electronic state of the dye molecules at the interface. Results of time-resolved measurements are presented in this paper, showing the electron injection can occur in less than 100 fs from the excited electronic state of dye molecules to empty states on the surface of the Lausanne-type anatase $\mathrm{TiO}_{2}$ electrode. This is shown in the rise of the product state, i.e. formation of the oxidized dye, and the decay of the reactant state, i.e. disappearance of the excited electronic state of the dye due to consumption in the interfacial reaction. The femtosecond timeresolved measurements were carried out on wet-chemistry prepared samples mounted in ultra-high-vacuum. The latter environment turned out to be necessary for completely eliminating irreversible side reactions of the perylene chromophore applied for these measurements ${ }^{11}$. The perylene chromophore was chosen since it offered many advantages compared to other dyes for obtaining unambiguous results with femtosecond time-resolved measuring techniques, i.e. transient absorption of the excited and oxidized state of the dye and fluorescence up-conversion.

Two important additional features were established for the injection reaction. Firstly, as expected ${ }^{12}$ this fast interfacial injection reaction was found independent of temperature, measured between $300 \mathrm{~K}$ and $20 \mathrm{~K}$. Secondly, the shorter than $100 \mathrm{fs}$ time constant for the injection reaction was found in the presence of a tunneling barrier for the electron corresponding to three saturated chemical bonds that were inserted between the rim of the chromphore and the nearest atom of the semiconductor electrode. Thus, contrary to what has been assumed by other investigators, direct coupling of the chromophore via a short chemical bond to the surface of the electrode is not required to make the injection reaction sufficiently fast. On the contrary, the width and height of the tunneling barrier can be utilized in principle as an important parameter for the optimum compromise between sufficiently fast electron injection and sufficiently slow electron-hole pair recombination. The experimental results also show that slow injection reactions with time constants in the ns time range, as reported by other authors for similarly prepared experimental systems ${ }^{13,14}$, cannot correspond to the molecular distances at the interface and energetic difference between donor and acceptor levels that were addressed in the present experiments. Slow electron transfer time constants in the ns range must correspond to much larger reaction distances than had been assumed for the respective wet-chemistry prepared interface.

The number of interfacial encounters with oxidized species and the respective molecular recombination times will determine the probability of injected electrons to reach the solid state contact at the base of the colloidal $\mathrm{TiO}_{2}$ electrode or suffer a recombination event. Three different recombination reactions can be distinguished at the electrochemical interface, i.e. geminate, non-geminate, and alien recombination ${ }^{15}$. 
These different recombination reactions have not yet been investigated in any detail in the dye-sensitized colloidal sponge-like $\mathrm{TiO}_{2}$ electrode. The Uppsala group described experiments ${ }^{16}$ showing that the injected electrons can escape over a certain distance from their place of origin. The essence of the observation is a certain escape distance for the injected electrons that is controlled by recombination events. However, a very simplistic model was applied by the Uppsala group in fitting the experimental results, i.e. pure diffusion was assumed as mode of transport for the injected electrons. The influence of a potential gradient and trapping events were neglected in the sponge-type $\mathrm{TiO}_{2}$ electrode. Basic considerations are presented here that these assumptions are in contradiction with the establishment of an equilibrium potential in the dark, and experimental results are presented that prove the dominance of multiple trapping and trap filling events for electron transport through the colloidal sponge-type anatase $\mathrm{TiO}_{2}$ electrode prepared by the Lausanne group.

\section{Results and discussion}

a) Time-resolved electron injection from excited dye molecules into the sponge-like anatase $\mathrm{TiO}_{2}$ electrode

Figure 1 illustrates the experimental system, and figure 2 shows the structure of the dye molecule employed for the present measurements. Note that electron transfer leads through three saturated bonds, or the equivalent distance through space, independent of the adsorption site, pocket in figure 2 , and of any conceivable change in the orientation of the anchored dye molecule. Anchoring was achieved via the dissociated phosphonate group. After adsorption from ethanolic solution the dye molecules

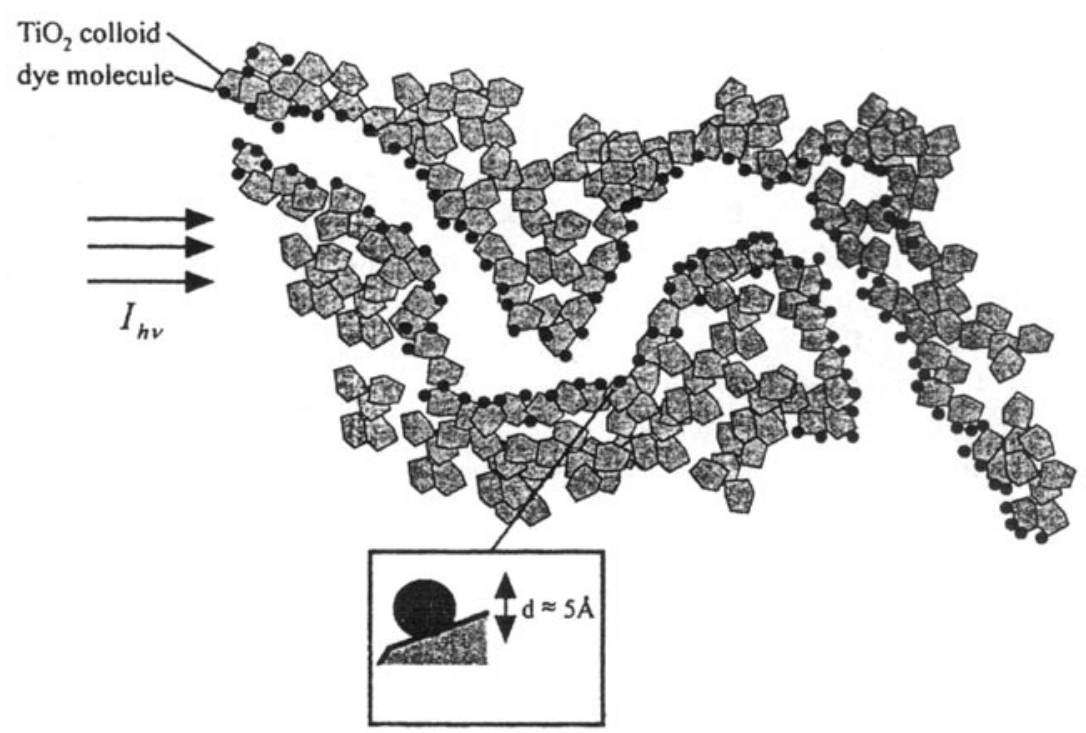

Figure 1. Illustration of the folded surface of the colloidal sponge-type $\mathrm{TiO}_{2}$ electrode with anchored dye molecules. After absorption of a photon interfacial electron transfer occurs from a dye molecule, black sphere, to the adjacent $\mathrm{TiO}_{2}$ colloid, grey particle. 


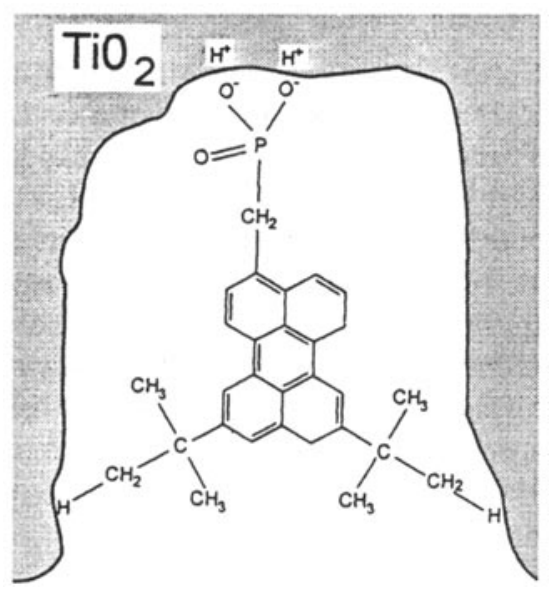

Figure 2. Illustration of the modified perylene chromophore anchored via a dissociated phosphonate group at the surface of the $\mathrm{TiO}_{2}$ electrode. Note that the bulky tetra-butyl groups guarantee that electron transfer proceeds via a tunneling barrier corresponding to three saturated bonds or the equivalent space, irrespective of the geometry of the adsorption site, a cavern is illustrated here, or any bending in the bonds of the side groups attached to the perylene chromophore.

remained anchored to the $\mathrm{TiO}_{2}$ electrode also when the latter was transferred to ultra-high-vacuum, as in the case of the experiments presented here. Details of the experimental apparatus are described elsewhere ${ }^{11}$. Figure 3 shows the transient absorption spectra of the oxidized state and the fluorescence up-conversion signal of the excited electronic state of the chromophore, respectively. The signals were measured in the appropriate time windows indicated as parameters with the dye molecules anchored on the surface of $\mathrm{TiO}_{2}$. Transient absorption was recorded at the peak wavelength of the absorption curve of $\mathrm{Pe}^{+}$shown in figure 3 , and the fluorescence up-conversion curve was measured close to the second peak of the dominant vibrational progression in the fluorescence spectrum, i.e. at $490 \mathrm{~nm}$ for the dye anchored at the sponge-like $\mathrm{TiO}_{2}$ electrode. The 0,0 -transition of the fluorescence spectrum could not be observed due to reabsorption of the fluorescence in the $4 \mu \mathrm{m}$ thick dye covered $\mathrm{TiO}_{2}$ electrode. The wide separation between the two spectra should be noted in figure 3. The disappearance of the reactant state, $\mathrm{Pe}^{\circ}$, and the rise of the product state, $\mathrm{Pe}^{+}$, are shown in figure 4 at the bottom and top, respectively. Both signals remained the same independent of temperature between $300 \mathrm{~K}$ and $20 \mathrm{~K}$. The insets give the respective instrumental functions for the two different types of measurements. The crosses in the curve at the bottom show a convoluted bi-exponential fit with the fast time constant of $190 \mathrm{fs}$ and the slow time constant 10 times longer. The transient absorption signal shown at the top contained a weak contribution due to a transient absorption response of the $\mathrm{TiO}_{2}$ electrode that will be described elsewhere. Correcting for the latter disturbance of the signal, the rise of the product state $\mathrm{Pe}^{+}$was fitted to a dominant time constant in the range of $70 \mathrm{fs}$. The faster rise of $\mathrm{Pe}^{+}$compared to the decay of $\mathrm{Pe}$ was tentatively ascribed to reabsorption and emission of fluorescence from dye molecules that were located at unfavorable sites for electron injection and thus with a longer lifetime with respect to charge injection. The time dependence of both the 


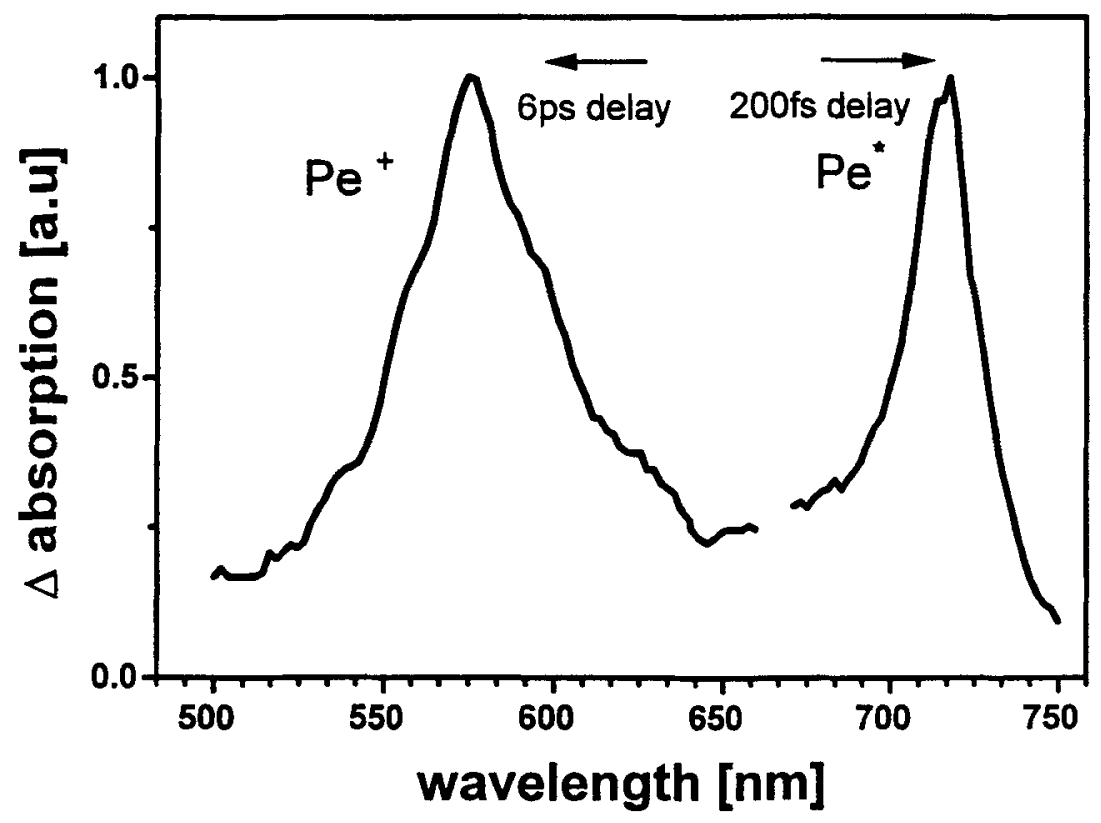

Figure 3. Absorption spectra of the excited electronic singlet state $\mathrm{Pe}^{*}$ and of the oxidized state $\mathrm{Pe}^{+}$of the modified perylene chromophore when anchored to the surface of the sponge-type anatase $\mathrm{TiO}_{2}$ electrode. Note the large spectral separation between the two absorption spectra. Two different time windows are indicated for recording the spectra due to the different life times of the respective electronic states $\mathrm{Pe}^{*}$ and $\mathrm{Pe}^{+}$, respectively.

experimental signals shown in figure 4 clearly established that electron transfer from the excited perylene chromophore occured in the range of $100 \mathrm{fs}$ through three saturated bonds or the equivalent space (figure 2).

It.should be noted that this electron transfer is much faster than is required for efficient electron injection. Considering the $5 \mathrm{~ns}$ lifetime of the excited singlet state of the perylene monomer in the absence of electron transfer quenching the desired injection probability of $99 \%$, corresponding to the branching ratio of the two competing rate constants, would be achieved already with an electron transfer time constant in the range of $50 \mathrm{ps}$, much longer than the $70 \mathrm{fs}$ in the present system. Considering a factor of 3 decrease for every saturated bond, the optimized spacer molecule would therefore comprise about 7 saturated bonds inserted between the chromophore and the semiconductor surface, compared to 3 saturated bonds in the present system. Optimization of the spacer length is desired since a longer spacer will slow down each of the three different recombination channels, i.e. geminate, non-geminate, and alien recombination between electrons and holes ${ }^{15}$, and it will thus reduce recombination losses that can occur at the interface.

Electron transfer spectra are illustrated in figure 5 for the simplest experimental situation, where electron transfer occurs from the vibrational ground state of $\mathrm{Pe}^{*}$. The shape of the electron transfer spectrum is controlled by the different Franck-Condon factors for the transition from the vibrational ground state of the reactant state $\mathrm{Pe}^{*}$ to the different vibrationally excited states of the product state $\mathrm{Pe}^{+}$, and to lesser degree 


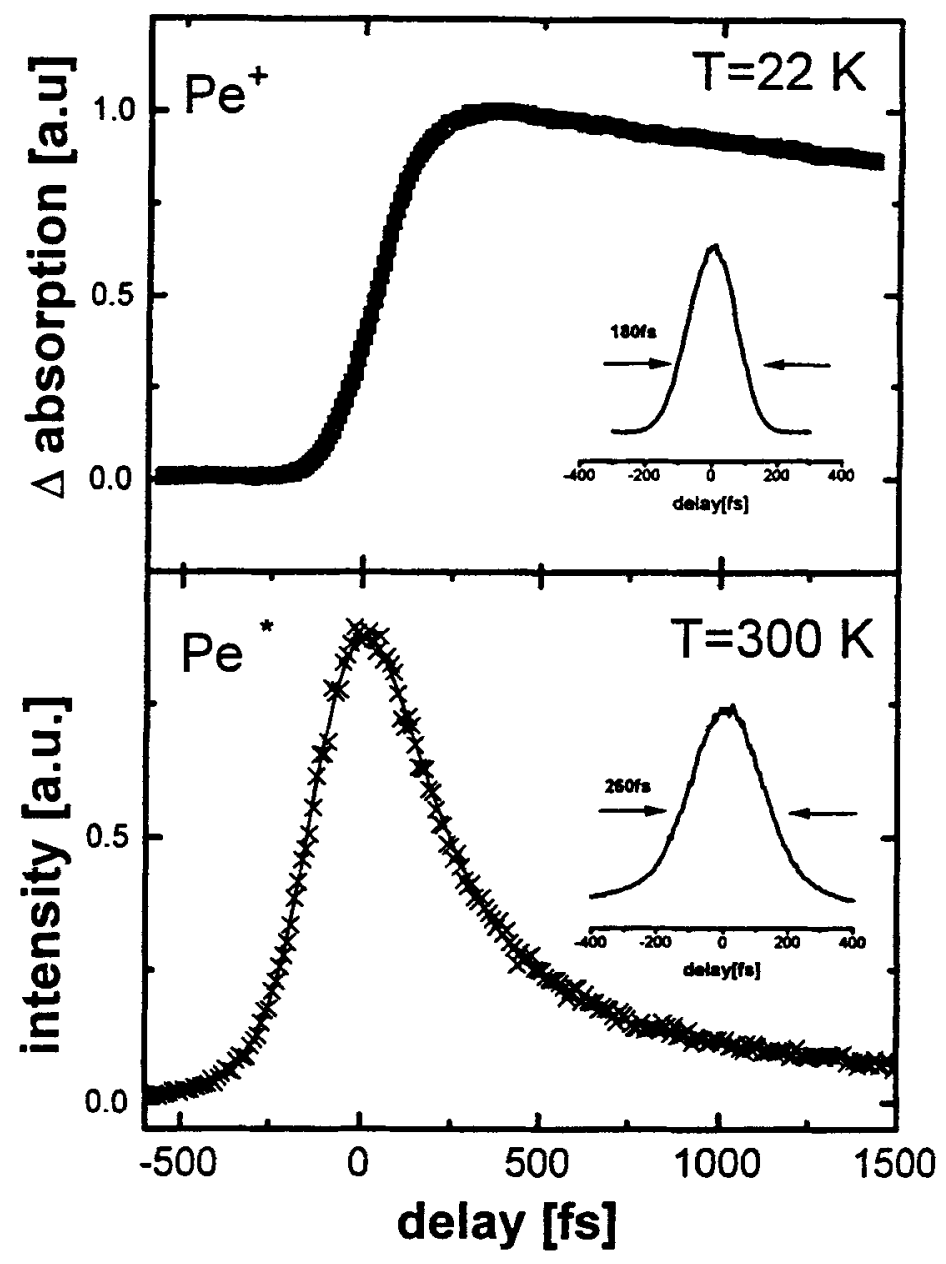

Figure 4. Time dependent decay of the excited singlet state $\mathrm{Pe}^{*}$ and rise of the oxidized state $\mathrm{Pe}^{+}$of the perylene chromophore anchored to the surface of the sponge-like anatase $\mathrm{TiO}_{2}$ electrode as measured in ultra-high-vacuum in the temperature range between $300 \mathrm{~K}$ and $22 \mathrm{~K}$. The upper signal corresponds to transient absorption measured at $580 \mathrm{~nm}$, the maximum of $\mathrm{Pe}^{+}$absorption (compare figure 3), and the lower signal corresponds to fluorescence up-conversion measured at $490 \mathrm{~nm}$, corresponding to the third vibrational peak of the fluorescence. The two insets illustrate the respective shape of the instrumental time response. The crosses in the lower curve show a biexponential fit to the convoluted signal.

by a possible change in the density of unoccupied electronic levels in the respective energy range in the $\mathrm{TiO}_{2}$ electrode. The spectrum on the left hand side corresponds to the probability of occupying vibrational excited states of the product state $\mathrm{Pe}^{+}$in the electron transfer reaction that starts at the vibrational ground state of the reactant $\mathrm{Pe}^{*}$ generated by the absorption of a photon with the 0,0 -energy of the transition from the ground state $\mathrm{Pe}$ to the excited state $\mathrm{Pe}$ * The spectrum at the right hand side stands for the probability of occupying an unoccupied electronic state with the respective energy 


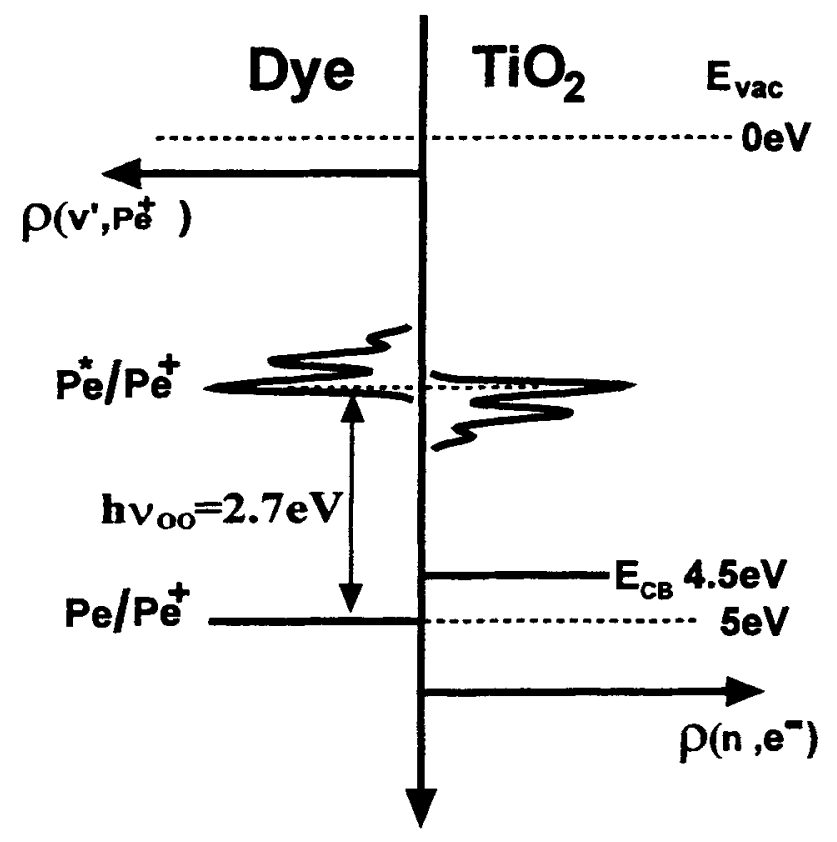

Figure 5. Illustration of the electron transfer spectra or distribution curves corresponding to electron transfer from the vibrational ground state of $\mathrm{Pe}^{*}$ : At the right hand side the probability of generating electrons, wavepackets with the respective energy shown in the conduction band of the semiconductor $\mathrm{TiO}_{2}$, at the left hand side the probability of generating different vibrationally excited states of $\mathrm{Pe}^{+}$. The distribution curve reflect the Franck-Condon factors for the electron transfer reaction from the vibrational ground state of $\mathrm{Pe}^{*}$ and are slightly modified by density of states effects in the conduction band of the semiconductor.

in the conduction band of $\mathrm{TiO}_{2}$. Energy conservation for isoenergetic electron transfer couples the generation of an electronic wavepacket at lower energy in the conduction band with the generation of a vibrationally excited state of $\mathrm{Pe}^{+}$of higher energy, shown closer to the zero energy level of the scale shown in figure 5. The measured temperature independence of the fast electron transfer signals shown in figure 4 confirms the expectation ${ }^{12}$ that the oxidation potential of the excited state $\mathrm{Pe}^{*}$ is located high above the lower edge of the conduction band, or lowest unoccupied electronic levels, $E_{C B}$ of the colloidal $\mathrm{TiO}_{2}$ electrode and that there are a high density of higher lying unoccupied electronic levels available for the electron transfer reaction. The high energetic position assumed for the oxidation potential of $\mathrm{Pe}^{*}$ in figure 5 would also allow for the generation of the excited electronic state of the oxidized dye molecule $\mathrm{Pe}^{+17}$. The positions of the energy levels given in figure 5 are estimates derived from photoelectron spectroscopy and from electrochemical data ${ }^{11}$. The dominant vibrational mode shown in the electron transfer spectrum was assumed to be identical to that found in the photoionisation spectrum of the chromophore perylene. From this latter spectrum and from data on the transport of charge carriers in the perylene single crystal, it can be deduced that the reorganisation energy or relaxation energy for going from $\mathrm{Pe}^{*}$ to $\mathrm{Pe}^{+}$is smaller than $0.1 \mathrm{eV}^{11}$. It should be noted that the structured electron 
transfer spectrum shown in figure 5 corresponds to the structureless Gaussian electron transfer spectrum that is often assumed for the so-called distribution curve of a redox species when performing a homogeneous or interfacial electron transfer reaction at room temperature ${ }^{18,19}$.

\section{b. Potential distribution and time-resolved transients of electron transport through the} colloidal sponge-type $\mathrm{TiO}_{2}$ electrode

One of the surprising properties of the dye-sensitized electron current flowing through the sponge-type $\mathrm{TiO}_{2}$ electrode was the high current efficiency close to $80 \%$ with respect to the incident photon flux ${ }^{8-10}$. It was discovered by Lindquist $e t$ al that this current collection efficiency decreased dramatically when the electrode thickness was increased and the light was impinging on the surface opposite to the electron collecting solid state contact, e.g. $\mathrm{SnO}_{2}: \mathrm{F}$. These authors ${ }^{16}$ introduced a finite escape length for the injected electrons in the form of a diffusion length. The numerical value for this diffusion length was derived from a simple diffusion model describing the transport of electrons away from their place of origin at one surface of a slab of material standing for the sponge-like $\mathrm{TiO}_{2}$ electrode illustrated in figure 1. A possible potential gradient was ignored in this slab of material, trapping and detrapping events were neglected, whereas recombination events at the folded interface of the sponge-like electrode were implictly introduced via the finite diffusion length. The intuitively convincing ingredient in this model was the assumption of a finite escape range for the injected electrons measured from their place of origin on one of the colloidal particles at the interface of the sponge-like $\mathrm{TiO}_{2}$ material with the enclosed solvent containing the redox ions (figure 1). This finite escape range should be controlled by recombination of the injected electron molecules at the interface, either with oxidized dye molecules or with oxidized redox ions. However, the other implicit assumptions in the simple diffusion model appear less satisfactory for obtaining a physically meaningful description of electron transport through this colloidal sponge-like electrode. Two of the fundamental properties of this electrode that are missing in the above model are considered in this section, i.e. the occurence of a potential gradient set-up in the dark, and multiple trapping, detrapping and trap filling events. Both these properties change the transport properties of electrons in this electrode when the stationary light level is changed, and thus the electron density is changed.

The relevant electronic levels of the different components of a corresponding electrochemical cell are shown in figure 6 prior to contact formation. For simplicity a colloidal slab material is assumed first as in the model of Lindquist et $a l^{16}$. The potential for the electrons in the system $E_{c}$ is shown in figure 7 for three different scenarios that arise for different assumptions about the concentration of donor doping in the $\mathrm{TiO}_{2}$ electrode and the speed of electron exchange at the semiconductor/electrolyte contact. At the top of figure 7 the equilibrium potential drop is illustrated for the case where the $\mathrm{TiO}_{2}$ electrode is n-type and develops a depletion layer at the contact with the electrolyte. In the center of figure 7 it is shown for the case where the $\mathrm{TiO}_{2}$ electrode is an insulator, and there is no exchange of electrons with the electrolyte in the dark. At the bottom of figure 7 the $\mathrm{TiO}_{2}$ electrode is also assumed to be an intrinsic insulator, but the equilibrium potential is established via injection of electrons from the redox electrolyte into the insulator electrode ${ }^{20}$. Note that the potential drop shown for the scenario depicted at the center of figure 7 is developed via 


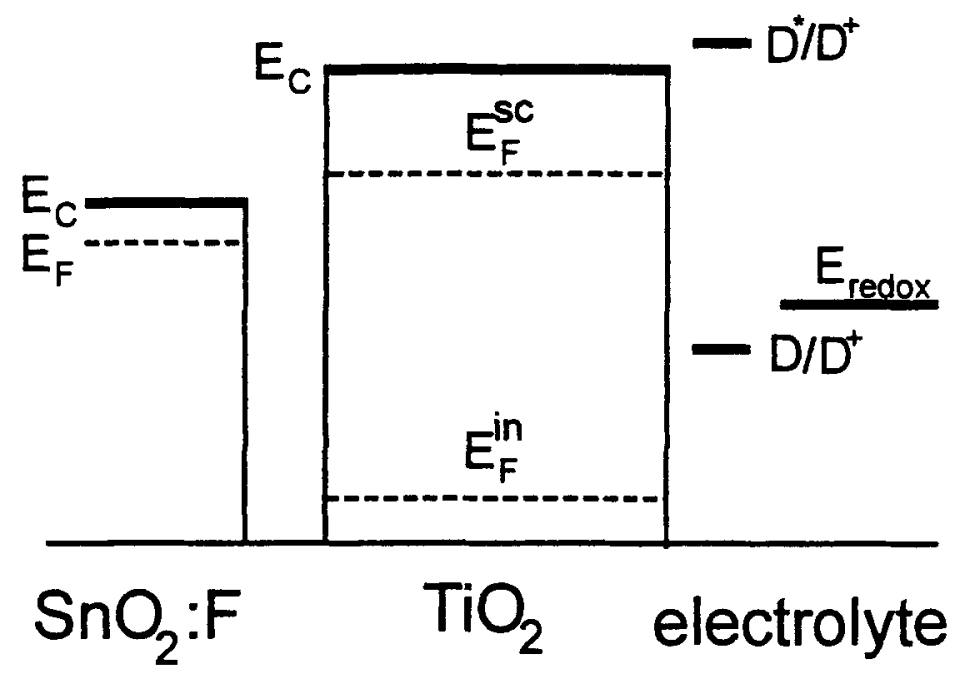

Figure 6. Illustration of idealized electronic levels in the different materials of the injection cell before contact formation: $E_{\text {redox }}=$ redox potential of the redox system in solution, $E_{D / D^{+}}, E_{D^{*} / D^{+}}=$standard redox potential for the oxidation of the dye molecules in the ground state $\mathrm{D}$, and excited electronic state $\mathrm{D}^{*}$, respectively. $E_{c}=$ conduction band, and $E_{F}=$ Fermi level for the broad band semiconductor, and for the solid state contact material. $E_{F S C}, E_{F i n}=$ respective Fermi level of the broad band semiconductor, when it is either doped with electron donors, or when it is an intrinsic insulating material.

transfer of electrons through the external load from the $\mathrm{SnO}_{2}: \mathrm{F}$ layer, with a higher lying Fermi level before contact formation, to the redox electrolyte. The system reaches equilibrium when the potential that is set-up across the insulator electrode brings the Fermi level at the left to the same energy level as the redox potential at the right of figure 6 . The scenario shown at the bottom appears the least likely for the system under consideration, since the energetic distance between the standard redox potential of the iodide/iodine couple that is usually applied for measuring the stationary photocurrent and photovoltage ${ }^{8-10}$, is more than $800 \mathrm{eV}$ away from the bottom of the conduction band of the $\mathrm{TiO}_{2}$. The energy difference is at least this large since $800 \mathrm{mV}$ were observed as open circuit voltage in the system ${ }^{1}$. The reorganisation energy is expected to be large for this redox couple also slowing down electron transfer in the system. The electron transfer time constant is expected in the range of many seconds to minutes for such a redox system at this uphill energy difference ${ }^{20}$. Thus, it should be slower than the RCcontrolled speed of electron transport through the external load considered for the scenario in the absence of electron exchange shown at the center of figure 7 . The two most likely scenarios are those shown at the top and in the center of figure 7. Both predict the development of a potential gradient in the dark that can drive electrons from the surface into the bulk of the electrode. The photopotential arising from electron injection by the excited dye, level $\mathrm{D}^{*} / \mathrm{D}^{+}$, would bring about a change in the potential towards the situation shown in figure 6. The sponge-like structure of the actual $\mathrm{TiO}_{2}$ electrode brings about some severe modifications but no basic difference arises with 


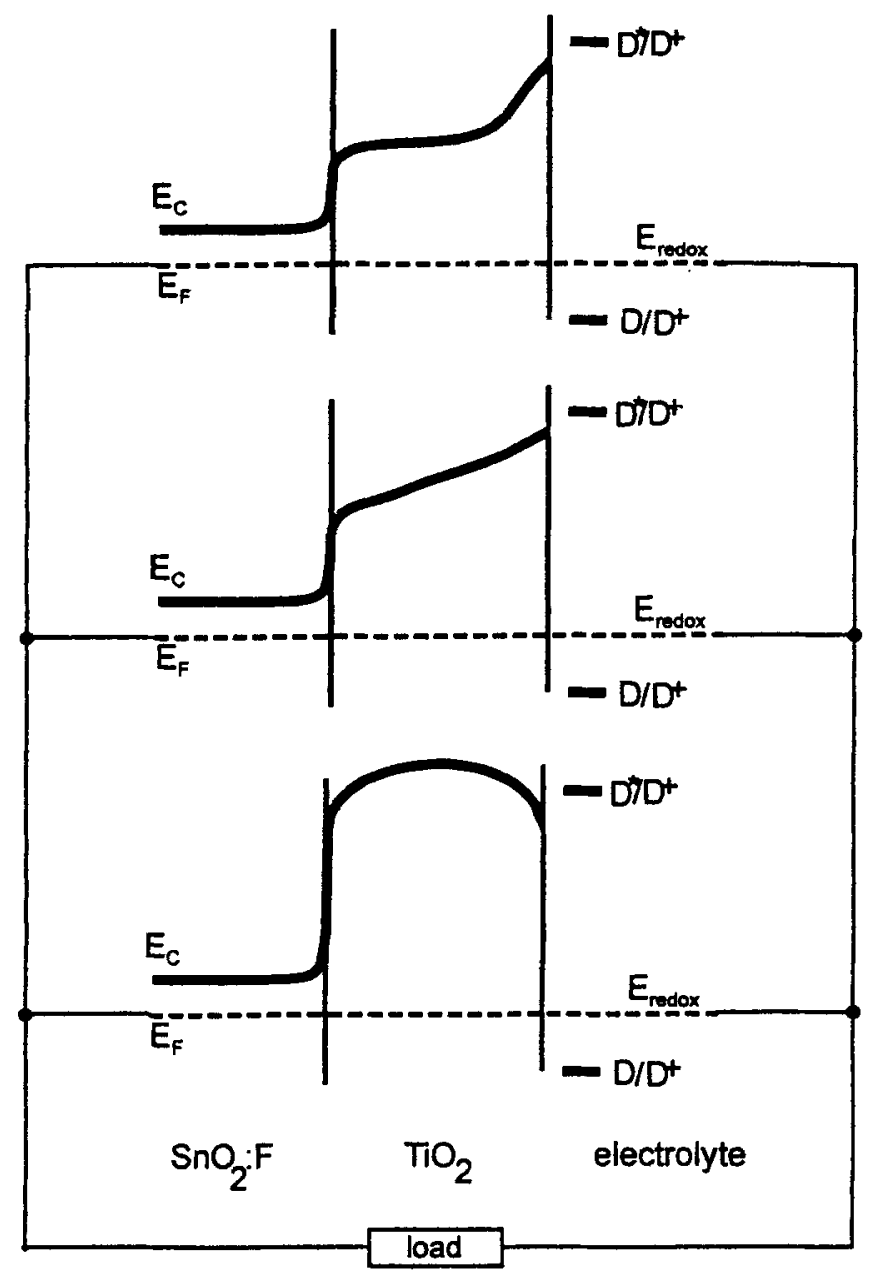

Figure 7. Illustration of the Fermi levels, $E_{F}$ and $E_{\text {redox }}$ respectively, and the conduction band energies $E_{c}$, in the injection cell after equilibrium is established in the dark. Three different cases are illustrated at the top, center, and bottom, respectively, corresponding to different types of charge exchange that can arise at the semiconductor/electrolyte interface. The load resistor is shown to illustrate that electronic contact formation is assumed between the two materials, solid state contact and electrolytic contact, for all three cases. Top: Semiconductor with a wide band gap and doped with electron donors, formation of a space charge due to electron transfer to the redox system leaving behind a space charge of ionized donors in the depletion layer. Center: Semiconductor with a wide band gap behaving as a true insulator in the dark, i.e. absence of charge exchange with the redox system in the dark. Charge exchange and equilibrium of the Fermi levels through the external load. Bottom: Semiconductor with a wide band gap behaving as a true insulator in the dark but with a sufficiently small separation between conduction band and standard redox potential that allows for the injection of electrons by the redox ions forming a mobile (in the absence of traps) space charge in the semiconductor. 
respect to the above considerations of the potential set-up in the dark. A representative geometry occuring in the sponge-type electrode is depicted in figure 8a. A cross section is shown through the long axis of a finger-type protrusion and through a layer of tightly packed colloids shown close to the solid contact material $\mathrm{SnO}_{2}$. The potential drops along the lines drawn in the grey colored collodial material of the electrode. Figure $8 \mathrm{~b}$ shows that the whole potential difference between $E_{F}$ and $E_{\text {redox }}$ in figure 6 drops either over one colloidal diameter corresponding to the tightly packed layer of colloids shown on top of the $\mathrm{SnO}_{2}$ layer in figure 8a, or over the much longer distance corresponding to the length of the protrusion shown in figure $8 \mathrm{a}$ and illustrated in figure $8 \mathrm{~b}$. Clearly, the sometimes applied comparison between the diameter of a single collodial particle and the width of a depletion layer at the surface of a slab of semiconductor material in contact with a redox electrolyte is meaningless for predicting the potential drop that arises in the colloidal sponge-type electrode. At the bottom of figure $8 \mathrm{~b}$ and in figure $8 \mathrm{a}$ it is seen that the potential gradient should drive the injected electrons towards the center of the finger-type protrusion and away from the surface in contact with the electrolyte. Thus, the correct consideration of the potential in the sponge-type $\mathrm{TiO}_{2}$ electrode is of great significance for assessing the average number of encounters of the injected electrons with oxidized dye molecules and with oxidized redox species at the interface to the electrolyte. This number and the reaction times decide whether the injected electrons can reach the $\mathrm{SnO}_{2}: \mathrm{F}$ contact, or whether they will recombine at the interface before reaching the contact. Thus, a realistic description of the potential in the $\mathrm{TiO}_{2}$ electrode is essential for assessing the recombination losses in the system. The actual geometric distribution of all the localized charges, screening of these charges by other ions in the solution, screening of charges trapped in the electrode material by the high dielectric constant of $\mathrm{TiO}_{2}$, all these contributions are considered to be of great importance for the potential gradient experienced by the injected electrons.

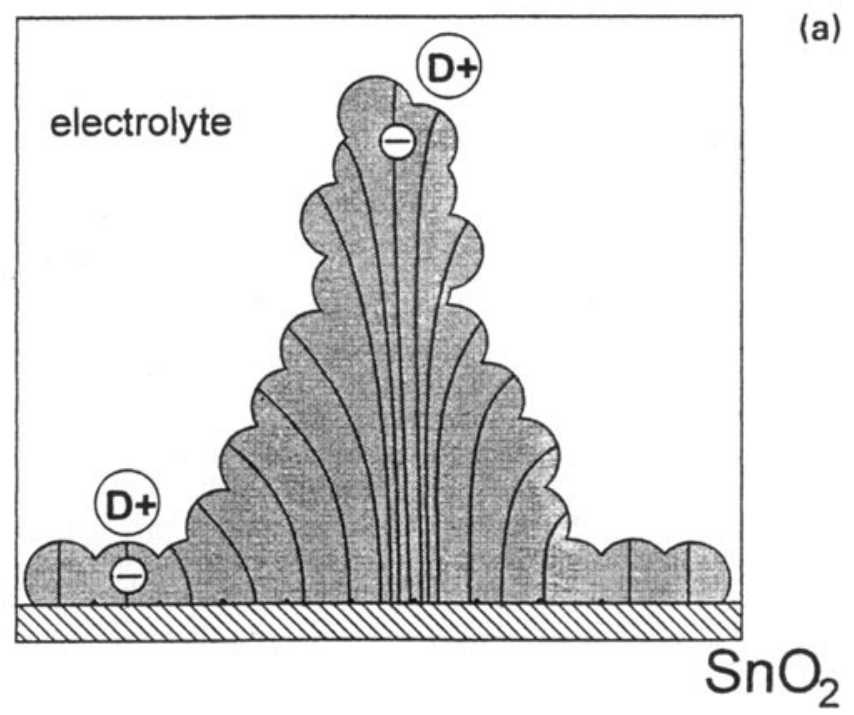

Figure 8. (a) 

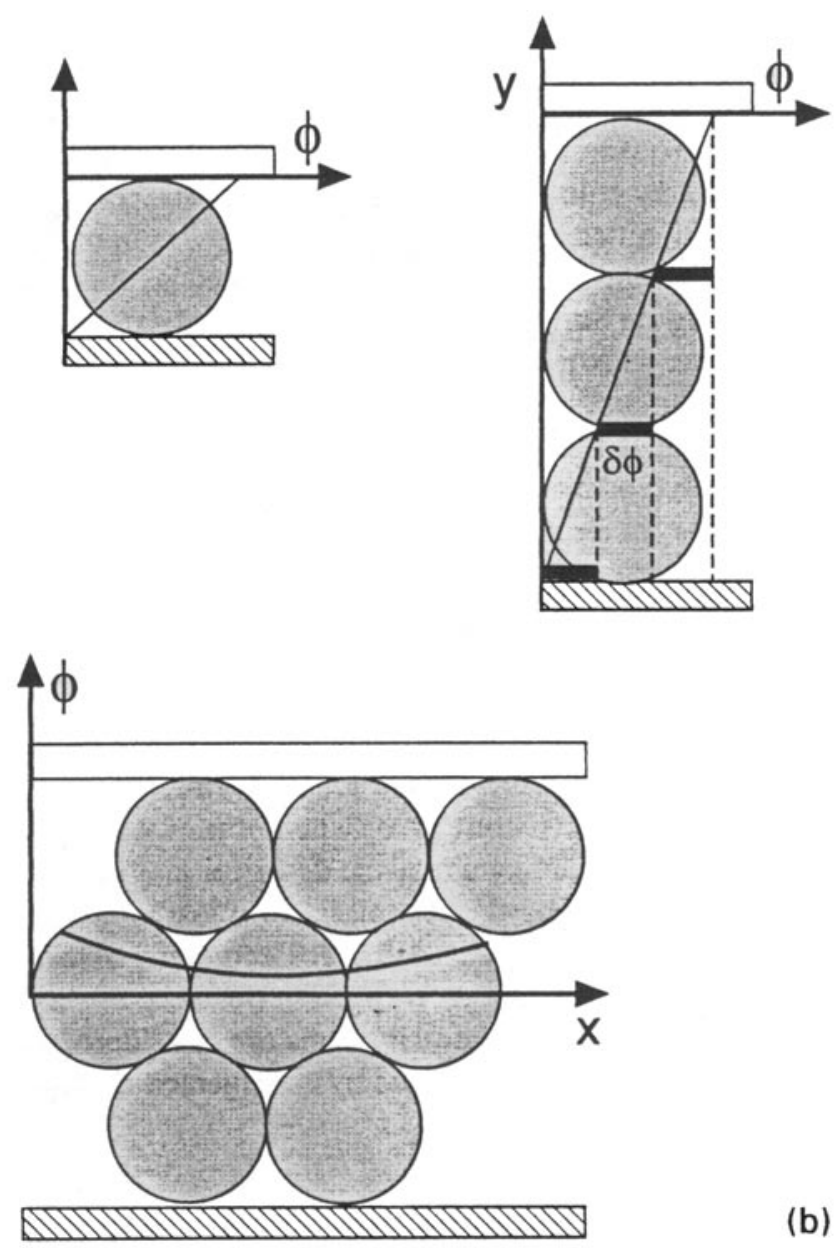

(b)

Figure 8. Electric field lines in a colloidal sponge-type electrode for the two cases illustrated at the top and in the center of figure 7.

a) Cross section of 3-dimensional configurations of the collidal $\mathrm{TiO}_{2}$ material that separate the electrolytic contact from the solid state contact $\left(\mathrm{F}: \mathrm{SnO}_{2}\right.$ in figure 7): Two extreme cases are illustrated here, i.e. a finger-like protrusion into the electrolyte and a tightly packed mono-colloidal layer on top of the solid state contact, respectively.

b) Top, left hand side: The potential difference drops over only one colloidal diameter corresponding to the thickness of the mono-colloidal layer between electrolyte and $\mathrm{SnO}_{2}: \mathrm{F}$ electrode. Top, right hand side: The same potential difference drops over many colloidal diameters along the long axis of the finger-type protrusion illustrated in a). Bottom: Potential developed across the diameter of the finger-type protrusion, i.e. in the direction perpendicular to the long axis of the finger-type protrusion illustrated in a). Note here that the potential gradient drives the injected electrons away from the electrolytic contact, i.e. firstly towards the center of the finger-type protrusion, and secondly towards the solid state contact $\left(\mathrm{F}: \mathrm{SnO}_{2}\right.$ in figure 7). 


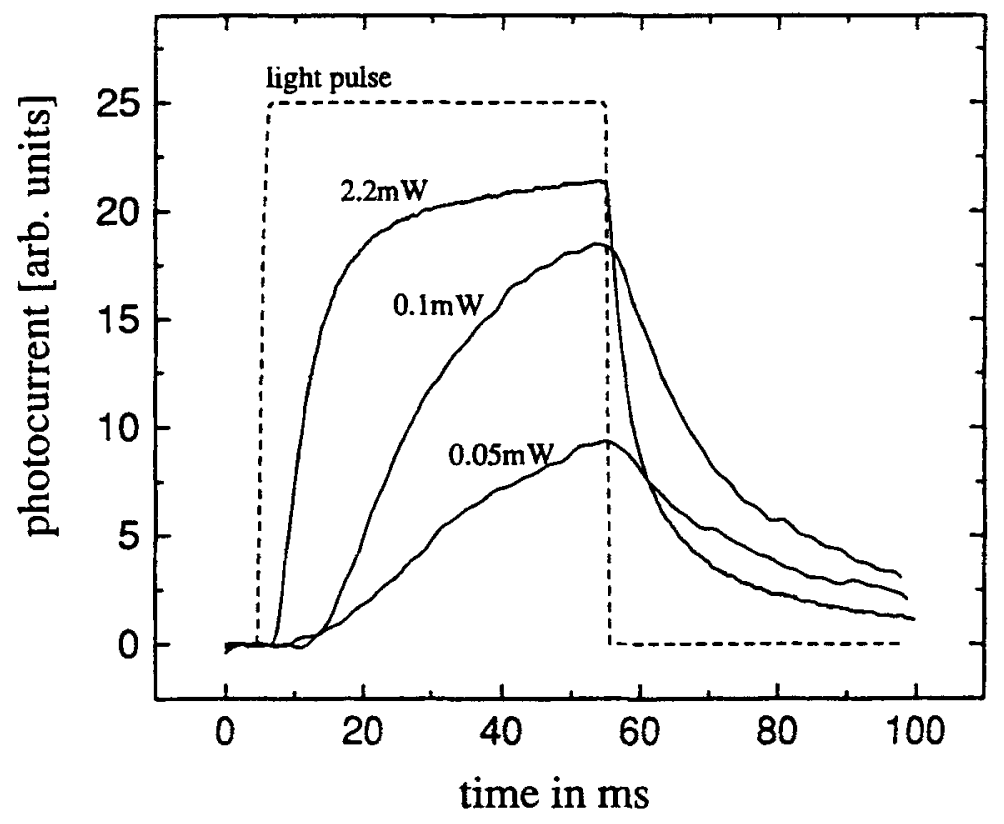

Figure 9. Electron transients measured with a 2-electrode configuration in the dye-sensitized colloidal sponge-type $\mathrm{TiO}_{2}$ electrode in response to a rectangular light-pulse. The three curves correspond to a different magnitude of the photon fiux that is given as parameter at the curves. Note the S-shape of the transients and the intensity dependent delay in the rise of the transients after the onset of the light pulse.

The electron transients measured at the colloidal sponge-type $\mathrm{TiO}_{2}$ electrode in the case of dye-sensitized injection (figure 9) duplicate all the important features that were already shown and discussed by two of the present authors for the Augustynski-type polycrystalline $\mathrm{TiO}_{2}$ electrode ${ }^{21}$. As analyzed before, the S-shape of the rising part of the transients and the change in the rise time with increasing photon flux (figure 9), parameter at the curves, prove that the filling of deep traps with injected electrons dominated the shape of the transients also in the colloidal sponge-type $\mathrm{TiO}_{2}$ electrode. Thus, trapping of injected electrons, delayed transport due to detrapping, and the non-linear trap filling kinetics ${ }^{21}$ must be essential ingredients of any model attempting to describe electron transport through this type of electrode. A realistic model for all the important processes must be developed to assess and influence the three different types of recombination losses ${ }^{15}$ that occur at the complicated interface of the $\mathrm{TiO}_{2}$ electrode with the adsorbed dye layer and adjacent electrolyte (figures 1 and 8).

\section{Acknowledgements}

The authors are grateful for financial support by the EU, Joule II program, and by the DFG, SFB 337. We thank Prof M Grätzel and Paul Liska, EPFL, for the gift of colloidal anatase $\mathrm{TiO}_{2}$ electrodes. 


\section{References}

1. Grätzel M 1996 paper presented at the IPS-11 conference, 28 July-2 August, 1996, Bangalore, India, and personal communication

2. For example, Nelson R C J. Opt. Soc. Am. 48 (1958); J. Phys. Chem. (1967) 71 2517; West W and Gilman P B 1977 in the Theory of the Photographic Process, 4-Edition, Ed. $\mathrm{T} \mathrm{H}$ James, MacMillan, New York and references therein

3. For example, Steketee J Wand de Jonge J 1963 Proc. Koninkl. Ned. Akad. Wetenschap. B66 76; Gerischer H, Michel-Beyerle M E, Rebentrost F and Tributsch H 1968 Electrochimica Acta 131509

4. The Theory of the Photographic Process, 1977, 4-Edition, Ed. T H James, MacMillan, New York

5. Trösken B, Willig F, Schwarzburg K, Ehret A and Spitler M T 1995 J. Phys. Chem. 995152

6. Pope M and Ch. Swenberg E 1982 Electronic processes in organic crystals, Oxford University Press, New York

7. Tsubomura H, Matsumura M, Nomura $Y$ and Amamiya T 1976 Nature 261402

8. Vlachopoulos N, Liska P, Augustynski J and Grätzel M $1988 \mathrm{~J}$. Am. Chem. Soc. 1101216

9. O'Regan B and Grätzel M 1991 Nature 353737

10. Nazeeruddin M K, Kay A, Rodicio I, Humphry-Baker R, Müller E, Liska P, Vlachopoulos N and Grätzel M 1993 J. Am. Chem. Soc. 1156382

11. Burfeindt B, Th. Hannappel, Storck W and Willig F J. Phys. Chem. accepted

12. Miller R J D, Mclendon G L, Nozik A J, Schmickler W and Willig F 1995 Surface Electron Transfer Processes, VCH, New York, chapter 5.

13. Hashimoto K, Hiramoto M, Kajiwara T and Sakata T 1988 J. Phys. Chem. 924636

14. Vinodgopal K, Hua X, Dahlgren R L, Lappin A G, Patterson L K and Kamat P V 1995 J. Phys. Chem. 99 10883; Argazzi R, Bignozzi C A, Heimer T A, Castellano F N and Meyer G J 1994 J. Inorg. Chem. 335741

15. Charlé K P and Willig F 1989 in Modern Aspects of Electrochemistry, Vol. 19, p., Eds. B E Conway, J Bockris, R E White, Plenum Press, New York p. 359

16. Södergren S, Hagfeldt A, Olsson J and Lindquist S E 1994 J. Phys. Chem. 985552

17. F W is grateful to Prof. J. Jortner for pointing out this possibility

18. Marcus R A 1965 J. Chem. Phys. 43679

19. Hopfield J J 1974 Proc. Nat. Acad. Sci. USA, 713640

20. Willig F 1981 in Advances in Electrochemistry and Electrochemical Engineering, Vol. 12, Eds. H Gerischer and Ch. W Tobias, Wiley, New York p. 1

21. Schwarzburg K and Willig F 1991 Appl. Phys. Lett. 582520 